American Journal of Applied Sciences 9 (6): 794-797, 2012

ISSN 1546-9239

(C) 2012 Science Publications

\title{
Clinical Pregnancy After Assisted Hatching Rate in Young women undergoing Intra Cytoplasmic Sperm Injection
}

\author{
Mohammed Al-Sunaidi \\ Department of Obstetrics and Gynecology, \\ College of Medicine, King Khalid University, Saudi Arabia
}

\begin{abstract}
Problem statement: The ability of a blast cyst to hatch, or escape, from the Zona Pellucida (ZP) that surrounds and protects the embryo during its first few days of development is one of many critical events that must occur for successful reproduction. Implantation of the embryo in the uterine lining is impossible unless hatching occurs. Hard or thick ZP will therefore impaire the implantation process and reduce the pregnancy rate. Approach: Retrospective analysis of patients' records who had ICSI cycles comparing pregnancy rate for women with and without assisted hatching between Jan and Dec 2010, this was to evaluate the overall effect of AH on pregnancy rates in younger women (less than 37 years old) undergoing ICSI cycles at Mustasharak medical center, ABHA, Saudi Arabia. The studied population was divided into four different subgroups according to age and the differences in clinical pregnancy rate among the assisted hatching group and control group were calculated. Results: Clinical pregnancy rate was found to be significantly higher statistically in young women who underwent laser assisted hatching after ICSI. Conclusion: Laser assisted hatching has a positive impact on the clinical pregnancy rate following ICSI in young age group.
\end{abstract}

Key words: Sperm injection, assisted hatching, assisted reproduction, zona pellucid, patients' records, clinical pregnancy, assisted reproductive, embryos transferred

\section{INTRODUCTION}

Intra Cytoplasmic Sperm Injection (ICSI), a technique allowing the microinjection of a single spermatozoon into the oocyte cytoplasm, has been applied worldwide to treat severe male factor infertility and dysfunction of fertilization. Although successful pregnancies have been achieved by ICSI, two thirds of embryos still fail to implant into a woman's uterus. Intrinsic abnormalities of the zona pellucida might be one of the causes for this implantation failure (Yang et al., 2012).

Increased maternal age, elevated basal FSH concentrations and poor embryo and zona pellucida morphology may also decrease an embryo's ability to hatch and implant following transfer (Wely and Veen, 2011). The ability of embryos to hatch from the zona pellucid is a crucial factor affecting the implantation process in assisted reproduction (Martins et al., 2011). Throughout nearly two decades, several experimental zona hatching techniques Assisted Hatching ( $\mathrm{AH}$ ) have been developed to improve the implantation and clinical pregnancy rates after IVF treatment (Martins et al., 2011; Ebner et al., 2005; Fleiss, 1981).
Using mechanical, chemical, or more recently implemented laser manipulations of the human zona pellucida, assisted hatching can be performed either by partial zona thinning, by drilling a hole through the zona, or by complete removal of the zona pellucida. Despite the vast number of published reports evaluating the use of assisted hatching for different clinical indications, the effectiveness of this procedure remains controversial (TPPAE, 2006). Published studies, most of them nonrandomized, have shown either no improvement in clinical outcome in the assistedhatching versus control groups or significantly increased implantation/pregnancy rates (TPPAE, 2006). Nonetheless, recent comprehensive reviews and metaanalyses of 23 prospective randomized clinical trials have suggested that assisted hatching may be beneficial for only a select group of patients, particularly those with repeated unexplained implantation failures and possibly older women (Seif et al., 2006) Existing trials provide insufficient data to determine the impact of assisted hatching on several important clinical outcomes, including live birth rates, monozygotic twining, congenital malformations and chromosomal abnormalities in children born from 
artificially hatched embryos (Martins et al, 2011; Mayor, 2010).

Although the specific indications for the use of assisted hatching remain unclear, the procedure is usually recommended for patients with poor pregnancy prognosis (TPPAE, 2006). But there is a lack of clinical studies on the impact of assisted hatching in the subpopulation of younger good prognosis patients.

The hypothesis of the study is that AH might overcome the deficiency of the zona pellucida and increase the implantation rate in ICSI cycles. However, at the same time it might allow the implantation of abnormal embryos. As a result, an increase in the live birth rate might not be observed because most embryos with chromosomal abnormalities would abort at an early stage of pregnancy. As with any technique in assisted reproduction, the long term consequences of ICSI remain an issue of concern. In particular, an increase in chromosome abnormalities (Tiboni et al., 2011; Woldringh et al., 2010) and an increase in imprinting disorders in ICSI-conceived newborns have been observed (Neelanjana and Sabaratnam, 2008). These findings might be due to the selected population undergoing fertility treatments or to the procedures themselves. A randomized evaluation of the outcome of pregnancies derived from combined ICSI and AH has yet to be reported.

The objectives of our study are to evaluate the overall effect of $\mathrm{AH}$ on pregnancy rates in younger women undergoing ICSI cycles.

\section{MATERIALS AND METHODS}

A retrospective study at Mustasharak assisted reproduction center, ABHA, Saudi Arabia, was performed for women records that underwent ICSI between Jan and Dec 2010. The study protocol was approved by the Institutional Review Board before beginning of the study and it was performed according to principles of Helsinki declaration.

Exclusion criteria for the study included failure of all eggs to be fertilized, poor quality embryos at the day of the transfer, or elective cryopreservation of all embryos. All study subjects underwent controlled ovarian hyperstimulation (with a long protocol regimen in all cases, including down-regulation with GnRH analogue and ovarian stimulation with recombinant FSH and transvaginal oocyte retrieval after the injection of 10,000 IU of HCG. ICSI was performed with the husband's spermatozoa according to the routine protocols at our center and sequential culture media from Vitrolife (IVF-20, G1.2 and G2.2, Scandinavian IVF Science AB, Göteborg, Sweden) were used in all cases.

Embryologists in the IVF lab evaluated the patients' embryos on day 3 after retrieval and the grading of each embryo was documented.

Laser AH was performed using the ZILOS-tk Zona Infrared Laser Optical System (Hamilton Thorne Instruments Biosciences, Beverly, MA) and embryos underwent laser zona ablation using 2-3 pulses (depending on thickness of the Zona Pellucida (ZP) at time of ablation) of 0.5-millisecond duration. The size of the hole made in the zona was measured to be 5-10 $\mu \mathrm{m}$, depending on the zona thickness of each individual embryo. A single operator was performing both ICSI and AH.

The primary outcome was clinical pregnancy, defined as the presence of fetal heart activity by ultrasound at 6-7 weeks' gestation.

Statistical analysis used Student's t test and chisquared test as applicable. Analysis of the data was performed using IBM® ${ }^{\circledR}$ SPSS ${ }^{\circledR}$ Statistics16 software.

\section{RESULTS}

A total of 381 patients' records were studied, 64 of them underwent laser assisted hatching. The studied sample was divided into four subgroups according to their age. Group A (age 20-25 years), group B (age 2530 years), group $\mathrm{C}$ (age $30-35$ years) and group $\mathrm{D}$ (age 36 37 years). A comparison of pregnancy rates between $\mathrm{AH}$ group and the control group was performed with $\mathrm{x}^{2}$ tests of independence and confidence intervals for the difference of two proportions. To assess the effect of maternal age, a similar analysis was used to compare the rates in the age group trials.

Table 1: Characteristics of patients' early follicular stage FSH and number of embryos transferred

\begin{tabular}{lcccccccccrrr}
\hline & Group A & & \multicolumn{3}{c}{ Group B } & \multicolumn{3}{c}{ Group C } & \multicolumn{3}{c}{ Group D } \\
& AH & Control & P value & AH & Control & P value & AH & Control & P value & AH & Control & P value \\
\hline *FSH & 5.1 & 6.0 & 0.38 & 5.7 & 5.2 & 0.15 & 5.3 & 5.4 & 0.23 & 5.4 & 5.3 & 0.76 \\
*No. ET & 3.2 & 3.8 & 0.19 & 3.5 & 3.6 & 0.20 & 3.5 & 3.2 & 0.25 & 3.5 & 3.2 & 0.20 \\
\hline
\end{tabular}

*The values represented are the mean. AH; Assisted Hatching, ET; Embryos Transferred 
Am. J. Applied Sci., 9 (6): 794-797, 2012

Table 2: Pregnancy rates in different age groups with and without AH

\begin{tabular}{|c|c|c|c|c|c|c|c|c|}
\hline & \multicolumn{2}{|l|}{ Group A } & \multicolumn{2}{|l|}{ Group B } & \multicolumn{2}{|c|}{ Group C } & \multicolumn{2}{|c|}{ Group D } \\
\hline & $\mathrm{AH}$ & Control & $\mathrm{AH}$ & Control & $\mathrm{AH}$ & Control & $\mathrm{AH}$ & Control \\
\hline Pregnancy rate & $50 \%$ & $39.9 \%$ & $65 \%$ & $47.4 \%$ & $49 \%$ & $39 \%$ & $50 \%$ & $38.7 \%$ \\
\hline Number of cases & 6 & 43.0 & 23 & 156.0 & 23 & 87 & 12 & 31.0 \\
\hline$*^{\mathrm{CI}}$ & $-0.53-0.35$ & $-0.40-0.04$ & $-0.3-0.16$ & $-0.4-0.23$ & & & & \\
\hline
\end{tabular}

*: $95 \%$ confidence interval. AH; Assisted Hatching

There were no significant difference between all the tested groups in term of the early FSH level and the number of embryos transferred as shown in Table 1.

The clinical pregnancy rate is found to be higher in assisted hatching group in all the different age groups tested and this was significant as shown by $95 \%$ confidence interval (Table 2).

\section{DISSCUSION}

In the current study we evaluated the impact of laser assisted hatching on clinical pregnancy rate in younger age group women who underwent ICSI for infertility treatment. We report a statistical significant improvement in pregnancy rate for young women who had ICSI treatment followed by laser assisted hatching compared with those who had ICSI alone.

The Zona Pellucida (ZP), which is made up of glycoprotein, is useful in maintaining embryo integrity. Before fertilization, the ZP surrounding the oocyte acts as a species-specific sperm barrier and is involved in sperm binding. After fertilization, the ZP plays a role in blocking polyspermic fertilization. It protects the integrity of the embryo and helps its oviductal transport. Elasticity and thinning of the $\mathrm{ZP}$ are fundamental for successful hatching. Before hatching occurs, the elasticity of the ZP enables the blastocyst to contract and expand, which decreases the thickness of the ZP (Hammadeh et al., 2011). Advanced maternal age and long-term exposure to suboptimal in vitro culture condition are associated with $\mathrm{ZP}$ hardening, possibly due to age-related changes or the absence of lysins from surrounding tissues, which may act on embryos in vivo (Suzumori and Sugiura-Ogasawara, 2010).

Although many studies have evaluated the effects of AH on implantation rates of embryos transferred after IVF, more than 15 years after the introduction of $\mathrm{AH}$ into the practice of assisted reproduction, the indications for its use still remain poorly defined.

Some studies have reported significant improvements in implantation rate and PR with the application of $\mathrm{AH}$ to treatment cycles by "poor prognosis" patients, defined by a history of repeated IVF-embryo transfer failures, elevated FSH, or advanced age (Toulis et al., 2010; Das et al., 2009). However, pooled analysis of such heterogeneous populations leaves ambiguity regarding whether all of these different reasons for a poor prognosis are responsive to $\mathrm{AH}$. A more recent randomized study reported no benefit for $\mathrm{AH}$ among patients classified as poor prognosis because of either advanced age or elevated FSH (Martins et al., 2011).

\section{CONCLUSION}

In conclusion, our study supports the findings by others that reported an age-related benefit after laser zona drilling. The need for large prospective randomized controlled trials is high to further evaluate the benefit of laser assisted hatching and to resolve the conflict.

\section{REFERENCES}

Yang, X.Y., J. Wang, J.Y. Liu, Y. Gao and Z.M. Zhou, et al., 2012. Pregnancy outcome after intracytoplasmic sperm injection with strontium oocyte activation in a globozoospermic patient. J. Asian. J. Androl. 14: 341-343. PMID: 22231301

Das, S., D. Blake, C. Farquhar and M.M. Seif, 2009. Assisted hatching on assisted conception (IVF and ICSI). Cochrane Database Syst Rev., 15: CD001894-CD001894. PMID: 19370570

Ebner, T., M. Moser and G. Tews, 2005. Possible applications of a non-contact $1.48 \mathrm{~mm}$ wavelength diode laser in assisted reproduction technologies. Hum. Reprod. Update, 11: 425-435.

Fleiss, J.L., 1981. Statistical Methods for Rates and Proportions. 2nd Edn., Wiley-Interscience, Hoboken, ISBN-10: 0471064289, pp: 321.

Hammadeh M.E., C. Fischer-Hammadeh and K.R. Ali. 2011. Assisted hatching in assisted reproduction: A state of the art. J. Assist. Reprod. Genet., 28: 119128. DOI: $10.1007 / \mathrm{s} 10815-010-9495-3$ 
Martins, W.P., I.A. Rocha, R.A. Ferriani and C.O. Nastri, 2011. Assisted hatching of human embryos: a systematic review and meta-analysis of randomized controlled trials. Hum. Reprod. Update, 17: 438-453. DOI: 10.1093/humupd/dmr012

Mayor, S., 2010. Risk of congenital malformations in children born after assisted reproduction is higher than previously thought. BMJ, 340: c3191-c3191. PMID: 20551127

Neelanjana, M. and A. Sabaratnam, 2008. Conditions in children born after assisted reproductive technology. Obstet. Gynecol. Surv. 63: 669-676. DOI: 10.1097/OGX.0b013e318181a9f0

Seif, M.M., E.C. Edi-Osagie, C. Farquhar, L. Hooper and D. Blake et al., 2006. Assisted hatching on assisted conception (IVF \& ICSI). Cochrane Database Syst Rev., 25: CD001894- CD001894. PMID: 16437437

Suzumori, N and M. Sugiura-Ogasawara, 2010.Genetic factors as a cause of miscarriage. Curr. Med. Chem., 17: 3431-3437. DOI: 10.2174/092986710793176302

Tiboni, G.M., I. Verna, F. Giampietro, E. Leonzio, G.G. Impicciatore, 2011. Cytogenetic findings and reproductive outcome of infertile couples referred to an assisted reproduction program. Gynecol. Endocrinol., $\quad 27$ : 669-674. DOI: $10.3109 / 09513590.2010 .533799$
Toulis K.A., D.G. Goulis, C.A. Venetis, E.M. Kolibianakis and R. Negro et al., 2010. Risk of spontaneous miscarriage in euthyroid women with thyroid autoimmunity undergoing IVF: A metaanalysis. Eur. J. Endocrinol., 162: 643-652. DOI: 10.1530/EJE-09-0850

TPPAE, 2006. Practice committee of the american society for reproductive medicine. Fertil Steril, 85: S18-S27. PMID: 17055818

Wely, M.V and F.V.D. Veen, 2011. To assist or not to assist embryo hatching. Hum. Reprod. Update, 17: 436-437. DOI: 10.1093/humupd/dmr019

Woldringh, G.H, D.E. Besselink, A.H. Tillema, J.C. Hendriks and J.A. Kremer, 2010. Karyotyping, congenital anomalies and follow-up of children after intracytoplasmic sperm injection with nonejaculated sperm: A systematic review. Hum. Reprod. Update, 16: 12-19. DOI: 10.1093/humupd/dmp030 